\title{
Effect of Implantation of Carbachol Pellet Near the Suprachiasmatic Nucleus on the Free-Running Period of Rat Locomotor Activity Rhythm
}

\author{
Tadashi Furukawa, Noboru Murakami, ${ }^{*}$ Kiyohisa Takahashi, ${ }^{* *}$ \\ and Teiichi ЕтоH \\ Department of Veterinary Physiology, Faculty of Agriculture, \\ University of Miyazaki, Miyazaki, 889 Japan \\ ** Division of Psychobiology, National Center for Nervous, \\ Mental and Muscular Disorders, \\ Kodaira, Tokyo, 187 Japan
}

\begin{abstract}
Summary To clarify the effect of a cholinergic agent on free-running period of circadian rhythm, locomotor activities were continuously recorded in the rat implanted with a paraffin pellet mixed with or without carbachol near the suprachiasmatic nucleus. Implantation of carbachol, but not paraffin alone, resulted in shortening of the free-running period under constant darkness.
\end{abstract}

Key words: circadian rhythm, suprachiasmatic nucleus, acetylcholine.

In many mammalian species, a light signal is mediated by the retinohypothalamic tract to the suprachiasmatic nucleus (MOORE and LENN, 1972) which has been proposed to be the site of the most predominant oscillator of the endogenous circadian rhythm (STEPHAN and ZUCKER, 1972; INOUYE and KaWAmUra, 1979; MOORE, 1983), and affects the phase or period of free-running rhythm (AsCHOFF, 1960; DAAN and PiTTENDRIGH, 1976). Recently, acetylcholine has been implicated in mediating the effects of light on the circadian system. Carbachol, a cholinergic agonist, mimics the phase-shifting effect or entraining effect of light on the circadian rhythm in rodents (ZATZ and HerKENHAM, 1981; EARNEST and TUREK, 1983). However, it is not yet clear whether acetylcholine is a certain neurotransmitter of the light pathway to the circadian system, since several agents other than acetylcholine have been also reported to cause a phase-dependent phase shift (Albers and FERris 1984; Albers et al., 1984).

If acetylcholine is the neurotransmitter related to the light effects, continuous

Received for publication November 17, 1986

* To whom all correspondence should be addressed. 
supply of carbachol to the suprachiasmatic nucleus may mimic the effect of constant light on the period of the free-running rhythm in rats (Honma et al., 1978; Summer et al., 1984). Previously we observed that implantation of carbachol near the suprachiasmatic nucleus shortened rather than lengthened the free-running period of rats, which was contrary to the effect of constant light on this nocturnal rodent (MuraKami et al., 1986). However, in that study, the free-running period was judged by acrophase of the water-intake rhythm determined at 4-h intervals once a week for 20 consecutive weeks. Such discontinuity of the measurement could lead to misinterpretation of the results, particularly concerning the phase of the freerunning rhythm. Therefore, we attempted to confirm the effect of carbachol implantation near the suprachiasmatic nucleus on the free-running period of the locomotor activity rhythm in the rat.

Adult male Spraque Dawley rats used in this study were bred in our laboratory and maintained under 14L:10D (lights on 0500-1900 h). Ten animals were transferred to a constant-dark room and to individual cages equipped with the measuring apparatus as previously reported (YoKAWA et al., 1986). The locomotor activities were counted in 30-min epoch by computer. The free-running period of the activity rhythm was determined by fitting a straight line through daily activity ends. The data were analyzed by a Student's $t$-test to evaluate the statistical significance of mean period of post-operation compared with that of pre-operation. About 3 weeks later, the rats were anesthetized with sodium pentobarbital, and paraffin pellets mixed with carbachol were bilaterally implanted near the suprachiasmatic nucleus. The paraffin pellets without carbachol were implanted as a control. The operation was performed at the resting period of animals. The midline of the earbars served as a reference for the anterior-posterior positioning. The stereotaxic coordinates for the pellet tubing were set at $6.4 \mathrm{~mm}$ rostal, $\pm 0.75 \mathrm{~mm}$ lateral from the midline, and $7.7 \mathrm{~mm}$ below the top surface of the skull. The preparation and implantation of the pellet were made by the method reported previously (MURAKAMI et al., 1983, 1986). Briefly, the carbachol pellet was made by kneading $10 \mathrm{mg}$ of carbochol powder uniformly into $100 \mathrm{mg}$ of paraffin at $55^{\circ} \mathrm{C}$. A punch prepared a $0.7 \mathrm{~mm}$-thick slab of this paraffin, and the pellet formed inside the punch (diameter $0.5 \mathrm{~mm}$ ) was injected into the animal, fixed with a brain stereotaxic apparatus, by means of a plunger fitted into the lumen of the punch. Each pellet weight was about $250 \mu \mathrm{g}$. About 5 or 15 weeks after implantation, the animals were perfused with $0.9 \%$ saline under sodium pentobarbital anesthesia, followed by $10 \%$ formalin. The implanted site was verified by microscopic examination of serial sections of the brain. In all except one control animal, the pellets had been implanted adjacently to the bilateral suprachiasmatic nucleus.

Although four rats were implanted with paraffin only as a control, locomotor activity patterns of all animals were not affected by the implantation of the pellet (Table 1, Fig. 1A), suggesting no effects of the pellet implantation, including surgical stress on the free-running rhythm. In this case, no significant difference was observed in the mean periods between pre- and post-implantation (Table 1). On the 
Table 1. Effect of carbachol implantation on the free-running period in the rat kept under constant darkness.

\begin{tabular}{cccccc}
\hline & \multicolumn{2}{c}{ Control (h) } & & \multicolumn{2}{c}{ Carbachol (h) } \\
\cline { 2 - 3 } \cline { 5 - 6 } & Pre-implant & Post-implant & & Pre-implant & Post-implant \\
\hline 1 & 24.51 & 24.51 & & 24.53 & 24.11 \\
2 & 24.57 & 24.57 & & 24.61 & 24.12 \\
3 & 24.62 & 24.62 & & 24.51 & 24.14 \\
4 & 24.66 & 24.66 & & 24.58 & 24.39 \\
5 & & & & 24.68 & 24.48 \\
Mean \pm S.E. & $24.59 \pm 0.03$ & $24.59 \pm 0.03$ & & $24.58 \pm 0.03$ & $24.25 \pm 0.08^{*}$ \\
\hline
\end{tabular}

Values are period of individual rats. Four control rats were implanted with paraffin alone. Post-implant is 5-week term after pellet implantation. $p$ vs. pre-implant $<0.01$.

other hand, the five of six rats implanted with carbachol pellet clearly showed shortening of the free-running period (Fig. 1B, C) during the following 5 weeks (Table 1). A remaining rat showed unclear activity pattern. When we continued to record the locomotor activities of two rats for more than 35 days after implantation, the free-running period observed before implantation was partially restored (Fig. 1C). However, complete restoration of the free-running period was not observed throughout the period examined for 100 days after implantation.

The present results and our previous observation (MURAKAMI et al., 1986) demonstrate that the implantation of carbachol near the suprachiasmatic nucleus causes the shortening of the free-running period in the rat. The free-running period returned toward the pre-treated value in about 5 weeks after implantation, which might indicate that the amount of carbachol released from the pellet decreased and became ineffective 5 weeks after implantation. Because, in the preliminary experiment, it is demonstrated that the releasing rate of ${ }^{14} \mathrm{C}$-acetylcholine from the pellet, which can be assumed to be the same as that of carbachol, remains constant for 4 weeks following implantation. It is well known that constant light causes lengthening of the free-running period in the rat (HonMA et al., 1978). In our rat colony, the mean free-running period is $25.67 \pm 0.05 \mathrm{~h}(n=6)$ under the constant light of about $300 \mathrm{~lx}$ and $24.58 \pm 0.06 \mathrm{~h}(n=6)$ in the constant darkness, respectively. In this respect, the effect of carbochol implantation on the free-running period is contrary to that of constant light. However, even under constant light, the impact of light upon the circadian pacemaker in the suprachiasmatic nucleus might vary, because light is frequently blocked by eyelids closed during sleep. On the other hand, implantation of carbachol near the suprachiasmatic nucleus may act constantly on the suprachiasmatic nucleus regardless of an active or resting condition of rat. Therefore, the effect of carbachol implantation cannot be simply compared with that of constant light. In the previous report, however, we observed that constant 

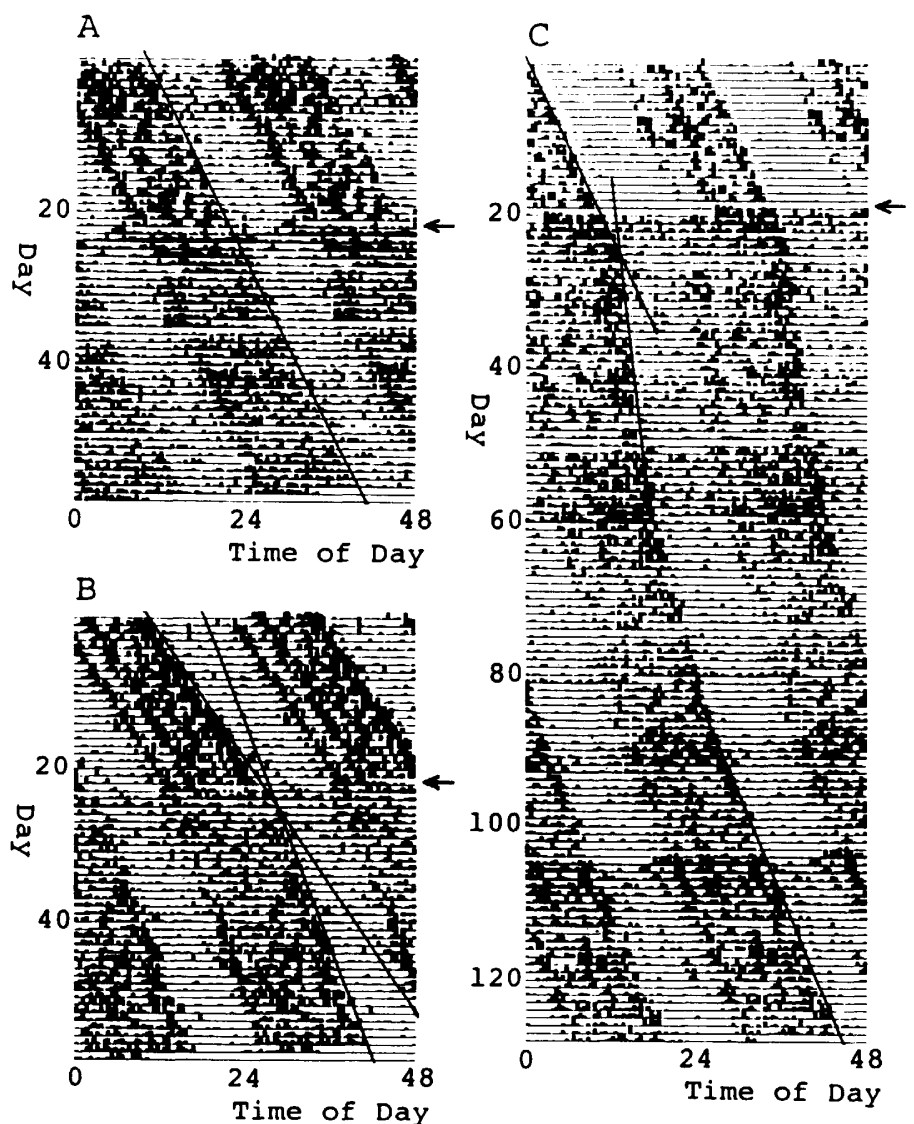

Fig. 1. Double plotted activity of three rats implanted with paraffin pellet (A), or pellet containing carbachol (B, C). Arrows indicate the day of implantation. Locomotor activity was recorded continuously and integrated at 30-min intervals by computer which drew histogram as activity counts as 30 -min period. The rats received histological examination of implant site, $5(\mathrm{~A}, \mathrm{~B})$ or $15(\mathrm{C})$ weeks after implantation.

light (about $5 \mathrm{~lx}$ ) causes shortening rather than lengthening of the free-running period in locomotor activity rhythm in eyelids-removed rats, but not in intact rats (MuraKami et al., 1986). The mean periods of free-running rhythm under constant light were $24.35 \pm 0.04 \mathrm{~h}(n=4)$ and $25.2 \pm 0.08 \mathrm{~h}$ in the eyelids-removed rats and intact rats, respectively. Although the removal of eyelids under constant light may not always bring about the constant impact of light upon the pacemaker in the suprachiasmatic nucleus, these results indicate that the effect of carbachol implantation is similar to the effect of constant light on the period in the rat without eyelids.

Intraventricular injection of carbachol has been shown to mimic the phase- 
shifting effect of light pulse on circadian rhythm of pineal enzyme activity in rats (ZATZ and BROWNSTEIN, 1979), of wheel-running activity in mice (ZATZ and Herkenham, 1981) and in golden hamsters (EARNEST and TUREK, 1985). In addition, intraventricular carbachol injection during the dark phase blocks the testicular regression in hamsters exposed to a short-day photoperiod in the same manner as light (EARNEST and TUREK, 1983). The present study indicates that carbachol implantation near the suprachiasmatic nucleus partially mimics the effect of constant light on the free-running period in eyelids-removed rats. These observations suggest that acetylcholine may be involved in some process of lightsignal transmission in the circadian system. However, contrary to this possibility it was reported that anticholinergic agents did not block the light-induced phase shift of locomotor activity rhythm in rats (PAULY and HorSEMAN, 1985) and than an antiGABAergic agent blocked the light-induced phase shift in the hamster (RALPH and MENAKER, 1985). Light increased the acetylcholine concentration of the suprachiasmatic nucleus with relatively slow time-course (MuraKami et al., 1984). Therefore, further studies are required to make the relationship between acetylcholine and light clear.

\section{REFERENCES}

Albers, H. E. and Ferris, C. F. (1984) Neuropeptide Y: Role in light-dark cycle entrainment of hamster circadian rhythms. Neurosci. Lett., 40: 163-168.

Albers, H. E., Ferris, C. F., Leeman, S. E., and Goldman, B. D. (1984) Avian pancreatic polypeptide phase shifts hamster circadian rhythms when microinjected into the suprachiasmatic region. Science, 223: 833-835.

Aschoff, J. (1960) Exogenous and endogenous components in circadian rhythms. Cold Spring Harbor Symp. Quant. Biol., 25: 11-28.

DaAn, S. and Pittendrigh, C. S. (1976) A functional analysis of circadian pacemaker in nocturnal rodents. II. The variability of phase response curves. J. Comp. Physiol., 106: 253-266.

Earnest, D. J. and TureK, F. W. (1983) A role for acetylcholine in mediating effects of light on reproduction. Science, 216: 77-79.

Earnest, D. J. and TuReK, F. W. (1985) Neurochemical basis for the photic control of circadian rhythms and seasonal reproductive cycles: Role for acetylcholine. Proc. Natl. Acad. Sci. U.S.A., 82: 4277-4281.

Honma, K., Katabami, F., and Hiroshige, T. (1978) A phase response curve for the locomotor activity rhythm of the rat. Experientia, 34: 1602-1603.

InOUYE, S. T. and Kawamura, H. (1979) Persistence of circadian rhythmicity in a mammalian hypothalamic "island" containing the suprachiasmatic nucleus. Proc. Natl. Acad. Sci. U.S.A., 76: 5962-5966.

MoORe, R. Y. (1983) Organization and function of a central nervous system circadian oscillator: The suprachiasmatic hypothalamic nucleus. Fed. Proc., 42: 2783-2789.

Moore, R. Y. and LenN, N. J. (1972) A retinohypothalamic tract in the rat. J. Comp. Neurol., 146: 1-14.

Murakami, N., Furukawa, T., Yokawa, T., Etoh, T., and Takahashi, K. (1986) Effects of continuous supply of light or carbachol to the SCN on the rat free-running rhythm. Jpn. J. Physiol., 36: 411-416. 
Murakami, N., Hayafuji, C., Sasaki, Y., Yamazaki, J., and Takahashi, K. (1983) Melatonin accelerates the reentrainment of the circadian adrenocortical rhythm in inverted illumination cycle. Neuroendocrinology, 36: 385-391.

Murakami, N., Takahashi, K., and Kawashima, K. (1984) Effect of light on the acetylcholine concentrations of the suprachiasmatic nucleus in the rat. Brain Res., 311: $358-360$.

Pauly, J. R. and Horseman, N. D. (1985) Anticholinergic agents do not block lightinduced circadian phase shifts. Brain Res., 348: 163-167.

RalPh, M. R. and MenaKer, M. (1985) Bicuculline blocks circadian phase delays but not advances. Brain Res., 325: 362-365.

Stephan, F. K. and ZuCKeR, I. (1972) Circadian rhythms in drinking behavior and locomotor activity of rats are eliminated by hypothalamic lesions. Proc. Natl. Acad. Sci. U.S.A., 69: 1583-1586.

Summer, T. L., Ferraro, J. S., and Mccormack, C. E. (1984) Phase-response and Aschoff illuminance curves for locomotor activity rhythm of the rat. Am. J. Physiol., 246: R299-R304.

Yokawa, T., Murakami, N., Furukawa, T., and Etoh, T. (1986) Effect of constant light on the circadian rhythm in locomotor activity in intact or eyelids-removed rat. Exp. Anim., 35: 185-188.

Zatz, M. and BRownstein, M. J. (1979) Intraventricular carbachol mimics the effects of light on the circadian rhythm in the rat pineal grand. Science, 203: 358-361.

Zatz, M. and Herkenham, M. H. (1981) Intraventricular carbachol mimics the phaseshifting effect of light on the circadian rhythm of wheel-running activity. Brain Res., 212: 234-238. 